\title{
Biological rhythms in reindeer
}

\author{
K.A. Stokkan ${ }^{1,3}$, B.E.H. Van Oort ${ }^{1}$ and N.J.C. Tyler ${ }^{2}$ \\ ${ }^{1}$ Department of Arctic Biology and Institute of Medical Biology, \\ ${ }^{2}$ Department of Biology, University of Tromsø \\ N9037 Tromsø, Norway
}

\begin{abstract}
Reindeer display numerous biological rhythms that prepare them for predictable changes in their environment. This form of biological timing generally results from an interplay between endogenous clocks and ambient signals of which the daily light-dark cycle is the most pronounced. Living at high latitudes, reindeer are exposed to extreme photic conditions throughout the year. As a consequence, they seem to have developed a form of biological timing which involves a reduction in the activity of endogenous clock mechanisms.
\end{abstract}

KEY WORDS: reindeer, biological clock, activity pattern, melatonin

The solar cycle creates a temporal framework of conditions to which plants and animals have adapted through responses known collectively as 'biological rhythms'. In reindeer (Rangifer tarandus) such rhythms have been recorded in both behaviour and physiological phenomena, such as the daily rhythm of activity and seasonal cycles of appetite and growth, fattening, moult, antler growth and reproduction. Biological rhythms are adaptive because they prepare the animals for predictable changes in the environment. Their regulation results from an interplay between endogenous "clocks" and exogenous time cues of which the 24 $\mathrm{h}$ light-dark (LD) cycle is the most prominent and ensures that particular functions are performed at the correct time of day or during the appropriate season. Such biological timing is believed to be a fundamental property of all organisms. It is phylogenetically very old but has characteristics that vary considerably, reflecting the life history of different species and the photic conditions to which they are normally exposed.

In mammals, the LD cycle is perceived via retinal photoreceptors to clockneurones in the hypothalamus. These neurones signal rhythmicity to the body

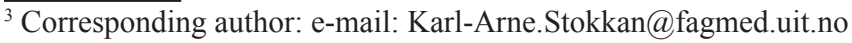


and, in particular, to the pineal gland making it secrete the hormone melatonin in a rhythmic, nocturnal pattern. The duration of secretion closely matches the duration of the night, thus, the pineal secretion of melatonin acts as a neuroendocrine transducer between the ambient LD cycle and the body.

Reindeer generally live in a highly seasonal environment and display pronounced biological rhythms which reveal the influence of biological timing. Living at high latitudes, reindeer are, moreover, exposed to photic conditions in which the LD cycle is virtually absent or markedly reduced for long periods each year. This occurs both during winter, when the sun remains permanently below the horizon, and during summer when it does not set. Such conditions are a potential challenge to the mechanisms of biological timing because, in general, continuous bright light wipes out the melatonin rhythm while permanent darkness leaves endogenous clocks unsynchronized.

In reindeer exposed to the continuous daylight conditions of summer at $70^{\circ} \mathrm{N}$, plasma melatonin concentrations remain permanently low (Eloranta et al., 1992; Stokkan et al., 1994) but begin to cycle with a $24 \mathrm{~h}$ period immediately following transfer of the animals into darkness. Prolonged exposure to the continuous bright light of midsummer seems to sensitise the clock to the progressive reduction in light intensity that occurs around midnight, inducing melatonin cycles and signalling autumn to the animals well in advance of a real shortening of the daylength. During the dark winter days, the titre of melatonin remains constant but at an elevated level and may also cycle depending, presumably, on local photic conditions. When transferred from LD conditions into permanent darkness, however, the rhythmic pattern of melatonin secretion disappears after a few cycles. Under natural photic conditions, free-ranging reindeer display daily rhythms of locomotor activity whenever there is a daily LD cycle. These do not, however, preclude a significant amount of nighttime activity. The daily rhythm of activity disappears during summer at $70^{\circ} \mathrm{N}$ and also in mid winter in reindeer living at $78^{\circ} \mathrm{N}$. At this high arctic latitude the daily pattern of activity appears less influenced by photic conditions even when there is a marked LD cycle.

Reindeer thus seem to have adapted to high-latitude photic conditions by a general reduction in the strength and activity of their endogenous clocks. One consequence of this is that the animals become opportunistically active around the clock during those periods each year when there is no marked day/night cycle.

\section{REFERENCES}

Eloranta E., Timisjärvi J., Nieminen M., Ojutkangas V., Leppälouto J., Vakkuri O., 1992. Seasonal and daily patterns in melatonin secretion in female reindeer and their calves. Endocrinology $130,1645-1652$

Stokkan K.A., Tyler N.J.C., Reiter R.J., 1994. The pineal gland signals autumn to reindeer (Rangifer tarandus tarandus) exposed to the continuous daylight of the Arctic summer. Can. J. Zool. 72, 904-909 